\title{
Influence of various substrates on the acetylcarnitine:carnitine ratio in motile and immotile human spermatozoa
}

\author{
R. Golan, D. P. Shalev, O. Wasserzug, R. Weissenberg and L. M. Lewin \\ Department of Chemical Pathology, Sackler Medical School, Tel Aviv University, Tel HaShomer, \\ and Department of Endocrinology, Sheba Medical Center, Tel HaShomer, Israel
}

\begin{abstract}
Summary. Human spermatozoa were incubated in albumin-containing Hepes-buffered modified Ringer's solution, in the presence or absence of externally supplied substrates. The acylated forms of carnitine were identified by bioautography. Incubation of the cells with propionate or $n$-valerate resulted in increased content of propionylcarnitine, but $n$-butyrate, isobutyrate, $n$-valerate, isovalerate, hexanoate or heptanoate did not result in the appearance of acylcarnitine of chain length $\mathrm{C}_{4}-\mathrm{C}_{7}$. The addition of methionine, valine or isoleucine (whose catabolic pathways should produce propionyl$\mathrm{CoA})$ to the incubation medium did not increase propionylcarnitine. In all cases acetylcarnitine was the major acylcarnitine in human spermatozoa.

The ratio of acetylcarnitine:carnitine remained relatively constant in spermatozoa incubated without external substrate for up to $4 \mathrm{~h}$. No significant change in the ratio was observed when glucose, fructose or citrate were present in the incubation medium. Sorbitol decreased the ratio slightly and aspartic acid slightly increased it. A more pronounced increase in the ratio was caused by lactate or pyruvate. This increase was observed in motile spermatozoa but not in samples from asthenospermic men, indicating that metabolic utilization of pyruvate and lactate may differ in motile and immotile cells.
\end{abstract}

\section{Introduction}

Carnitine is believed to play an important role in sperm metabolism because it is present in high concentration in spermatozoa and seminal fluid (Brooks, 1979), it is actively incorporated into spermatozoa during their maturation in the epididymis (Casillas, 1973), carnitine acyltransferase enzymes are found in high activity in spermatozoa (Brooks, 1979) and sperm mitochondrial metabolism can be affected by addition of carnitine to the incubation medium of permeabilized cells (Hutson, Van Dop \& Lardy, 1977). A role for carnitine in sperm motility has been suggested based upon the observation that spermatozoa first come into contact with significant concentrations of carnitine in the epididymal lumen at about the location in which they develop the capacity for progressive motility (Hinton, Brooks, Dott \& Setchell, 1981).

Addition of carnitine to immotile spermatozoa that had been removed from the proximal caput epididymidis of the rat caused these cells to start moving (Hinton et al., 1981). Other experiments have indicated that the acylation state of carnitine may be correlated with the ability of spermatozoa to move. Johansen \& Bøhmer (1979) and Golan, Weissenberg \& Lewin (1984) have demonstrated that the degree of acetylation of carnitine is greater in motile than in immotile human spermatozoa. Tanphaichitr (1977) and Jeulin, Soufir \& Jouannet (1981) have reported that addition of acetylcarnitine to ejaculated human spermatozoa stimulated motility slightly.

The experiments described here were to investigate the effects of various substrates on the acetylation state of carnitine in washed human spermatozoa incubated in a defined medium. 


\section{Materials and Methods}

Carnitine acetyltransferase, acetylcoenzyme $\mathrm{A}$ and DL-carnitine chloride were from Sigma Chemical Co., St Louis, MO, U.S.A. Acetylcarnitine, propionylcarnitine, isobutyrylcarnitine, and isovalerylcarnitine were generous gifts from Professor L. L. Bieber, Department of Biochemistry, Michigan State University, MI, U.S.A. The carnitine-requiring mutant yeast, Torulopsis bovina ATCC 26014, was obtained from the American Type Culture Collection, Rockville, MD, U.S.A. $\left[1-{ }^{14} \mathrm{C}\right]$ Acetylcoenzyme A was from the Radiochemical Centre, Amersham, U.K. Ficoll 400 was obtained from Pharmacia Corp., Uppsala, Sweden.

Semen samples were obtained by masturbation after 3 days of abstinence from patients attending a male infertility clinic at a general hospital. Measurement of sperm numbers and assessment of their morphology, motility and viability were made using routine procedures (Freund \& Peterson, 1976). Sperm samples showing greater than $50 \%$ good progressive motility and sperm counts greater than $40 \times 10^{6}$ cells $/ \mathrm{ml}$ were selected for studies of motile spermatozoa in contrast to asthenospermic samples in which at least $90 \%$ of the cells were living (as determined by eosin staining) but in which fewer than $10 \%$ were motile.

Washed spermatozoa were obtained by a modification of the method of Harrison (1976). Semen was layered on top of a solution of $10 \%$ Ficoll 400 in $0.9 \%(\mathrm{w} / \mathrm{v}) \mathrm{NaCl}$ in a conical centrifuge tube and centrifuged for $20 \mathrm{~min}$ at $2000 \mathrm{~g}$. The supernatant fluid was discarded and the sperm pellet was washed by resuspension in $0.9 \%(\mathrm{w} / \mathrm{v}) \mathrm{NaCl}$. The washed cells were then precipitated by centrifugation and resuspended to $10^{7}$ cells $/ \mathrm{ml}$ in glucose-free Hepes-buffered Ringer's solution supplemented with $1 \%$ bovine serum albumin as described by Gorus, Finsy \& Pipeleers (1982). In some experiments the mineral salts solution of Casillas \& Erickson (1975) was substituted for the medium described above. To determine the effects of addition of substrates, the medium was supplemented (e.g. with fructose, glucose, lactate, or pyruvate) to $10 \mathrm{~mm}$ concentration. The sperm suspensions $(2 \mathrm{ml})$ were incubated at $37^{\circ} \mathrm{C}$ for periods of $10 \mathrm{~min}$ to $4 \mathrm{~h}$, after which the activity of the carnitine acetyltransferase was inactivated. Three methods were tested for enzyme inactivation and for extraction of carnitine and acylcarnitines. Washed sperm pellets were used. In the first method the cells were chilled in ice and mixed with $2 \mathrm{ml}$ ice-cold perchloric acid $(6 \% \mathrm{w} / \mathrm{v})$, held for $10 \mathrm{~min}$ in ice, centrifuged for $10 \mathrm{~min}$ at $2000 \mathrm{~g}$ to precipitate proteins (which were discarded), and the supernatant liquid was neutralized with $\mathrm{KHCO}_{3}$ to $\mathrm{pH} 7 \cdot 0$ with bromthymol blue indicator. The potassium perchlorate precipitate was removed by centrifuging and the supernatant liquid was kept for assay of carnitine and its acyl esters. In the second method, the spermatozoa were frozen and thawed 3 times by placing the tubes alternately in a solid $\mathrm{CO}_{2}$-acetone bath and in tepid water, and were then extracted with absolute ethanol ( $1 \mathrm{ml}$ per $10^{7}$ cells). The third method consisted of placing the sperm pellet in a boiling water bath for $2 \mathrm{~min}$, followed by extraction with absolute ethanol $\left(1 \mathrm{ml}\right.$ per $10^{7}$ cells $)$.

Duplicate aliquants of the extracts (equivalent to $10^{7}$ cells) were dried at $60^{\circ} \mathrm{C}$. One of each pair was assayed for free carnitine by the method of Cederblad \& Lindstedt (1972). To the other sample $0.5 \mathrm{ml}$ of a concentrated solution of ammonium hydroxide $(33 \%)$ was added and the tube incubated at $37^{\circ} \mathrm{C}$ for $1 \mathrm{~h}$ to hydrolyse short-chain acylcarnitines. The ammonia was removed by evaporating to dryness and the total carnitine was then determined. The difference between total and free carnitine was taken as the acylcarnitine content of the samples.

Carnitine and its derivatives were separated by descending chromatography on Whatman No. 1 paper using the solvent system, $n$-butanol:acetic acid (glacial):water (8:1:1 by vol.). Chromatographic zones corresponding to the $R_{\mathrm{f}}$ values of standard derivatives were detected by bioautographic methods developed in our laboratory (Lewin \& Bieber, 1979; Lewin, Orenstein, Nebel \& Emaus, 1981), as follows: paper chromatograms were placed on transparent agar plates, seeded with a carnitine-requiring yeast strain (Torulopsis bovina ATCC 26014) and containing a carnitine-deficient nutrient medium. After incubation for $16 \mathrm{~h}$ at $37^{\circ} \mathrm{C}$ the paper chromatograms 
Table 1. A comparison of perchloric acid deproteinization, freeze-thawing treatment, and boiling as methods for inactivation of carnitine-acetyl transferase and extraction of carnitine and acetylcarnitine (pmol/106 spermatozoa).

\begin{tabular}{llccccc}
\hline Exp. & Method & $\begin{array}{c}\text { No. of } \\
\text { samples }\end{array}$ & L-Carnitine & $\begin{array}{c}\text { Total } \\
\text { carnitine }\end{array}$ & $\begin{array}{c}\text { Acetyl- } \\
\text { carnitine }\end{array}$ & $\begin{array}{c}\text { Acetyl- } \\
\text { carnitine:carnitine }\end{array}$ \\
\hline I & Freeze-thaw & 5 & $339 \pm 179$ & $1105 \pm 611$ & $766 \pm 463$ & $* 2 \cdot 38 \pm 1 \cdot 12$ \\
I & Perchloric acid & 5 & $461 \pm 102$ & $928 \pm 398$ & $474 \pm 323$ & $* 0 \cdot 99 \pm 0 \cdot 50$ \\
II & Freeze-thaw & 4 & $153 \pm-64$ & $822 \pm 286$ & $688 \pm 244$ & $4 \cdot 9 \pm 2 \cdot 5$ \\
II & Boiling & 4 & $153 \pm-73$ & $791 \pm 319$ & $637 \pm 268$ & $4 \cdot 8 \pm 2 \cdot 8$ \\
\hline
\end{tabular}

Values are mean \pm s.d.

${ }^{*} P<0 \cdot 05$, by $t$ test of difference between means.

were removed from the agar surface and growth zones at $R_{\mathrm{f}}$ values corresponding to L-carnitine and its derivatives were observed.

\section{Results}

\section{Enzyme inactivation and extraction of carnitine and acylcarnitines}

When spermatozoa were subjected to the 3 procedures, (1) inactivation with perchloric acid, (2) freeze-thawing of the cells, followed by ethanol extraction, and (3) inactivation at $100^{\circ} \mathrm{C}$ followed by ethanol extraction perchloric acid extraction led to lower acetylcarnitine:carnitine ratios than did the other two methods, which were in good agreement with each other (Table 1). The perchloric acid extraction method apparently resulted in partial hydrolysis of the acetylcarnitine. Method 3, which is simple to perform, was therefore selected for further use.

\section{Effect of medium on sperm motility and carnitine acylation}

The medium of Casillas \& Erickson (1975) did not support good progressive motility of human spermatozoa. The addition of bovine serum albumin (to $1 \%$ ) improved motility in this medium. Supplementation of Casillas' medium with fructose $(10 \mathrm{~mm})$ increased the acetylcarnitine:carnitine ratio significantly (by $56 \%, P<0.02$ ) when albumin was absent in the medium but not in its presence.

Additional experiments established that human spermatozoa from samples with good initial motility were capable of progressive motility for periods of more than $4 \mathrm{~h}$ when incubated in a glucose-free Hepes-buffered Ringer's solution, as described in 'Materials and Methods'. The ratio of acetylcarnitine:carnitine remained relatively constant during this period, during which motility was supported by endogenous reserves in the absence of exogenous substrate (Table 2). This medium was selected for the remaining studies. The effect of addition of various substrates on carnitine acylation was tested after incubation $(1 \mathrm{~h})$ at $37^{\circ} \mathrm{C}$.

\section{Effect of substrates on acylcarnitine composition}

Sperm extracts of cells that had been incubated without added external substrate were shown by bioautography to contain acetylcarnitine and free carnitine with only traces of propionylcarnitine. Addition of sodium salts of propionate or of $n$-valerate resulted in increased amounts of propionylcarnitine. Incubation of cells with $n$-butyrate, isobutyrate, $n$-valerate, isovalerate, hexanoate or heptanoate did not result in the appearance of acylcarnitines of 4-7 carbon chain lengths in sperm 
Table 2. Stability of acetyl carnitine:carnitine ratios in human spermatozoa in basal medium without added substrates

\begin{tabular}{ccccc}
\hline & \multicolumn{4}{c}{ Acetylcarnitine:carnitine } \\
\cline { 2 - 5 } Minutes & Exp. 1* & Exp. 2* & Exp. 3† & Exp. 4† \\
\hline 0 & $3 \cdot 0$ & $6 \cdot 5$ & $1 \cdot 9$ & $1 \cdot 5$ \\
30 & - & - & $2 \cdot 3$ & $2 \cdot 1$ \\
60 & $3 \cdot 4$ & $6 \cdot 1$ & $2 \cdot 1$ & $1 \cdot 8$ \\
90 & - & - & $2 \cdot 1$ & $3 \cdot 1$ \\
120 & $3 \cdot 2$ & $5 \cdot 5$ & $1 \cdot 8$ & $2 \cdot 1$ \\
150 & - & - & $2 \cdot 9$ & - \\
180 & - & - & $1 \cdot 8$ & - \\
210 & - & - & $2 \cdot 3$ & - \\
240 & $2 \cdot 8$ & $5 \cdot 3$ & $2 \cdot 5$ & - \\
270 & - & - & $2 \cdot 1$ & - \\
\hline
\end{tabular}

* Incubation at $37^{\circ} \mathrm{C}$, inactivation by boiling.

$\dagger$ Incubation at $30^{\circ} \mathrm{C}$, inactivation by freeze-thaw.

Table 3. The effects of incubation in the presence of pyruvate, sorbitol or citrate on the concentration (pmol/ $10^{6}$ spermatozoa) of carnitine and acetylcarnitine in human spermatozoa

\begin{tabular}{lcccccc}
\hline & L-Carnitine & $\begin{array}{c}\text { Total } \\
\text { carnitine }\end{array}$ & $\begin{array}{c}\text { Acetyl- } \\
\text { carnitine }\end{array}$ & $\begin{array}{c}\text { Acetyl- } \\
\text { carnitine: } \\
\text { carnitine }\end{array}$ & AAC:C* & $\begin{array}{c}\% \\
\text { Difference } \dagger\end{array}$ \\
\hline Control & 375 & 1164 & 790 & $2 \cdot 11$ & - & - \\
$\begin{array}{l}\text { pyruvate } \\
\text { + sorbitol }\end{array}$ & 242 & 1151 & 909 & $3 \cdot 80$ & $1 \cdot 69$ & 80 \\
+ citrate & 371 & 1093 & 723 & $1 \cdot 95$ & $-0 \cdot 16$ & $-7 \cdot 6$ \\
\hline
\end{tabular}

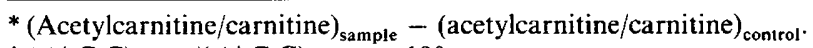

$\dagger(\Delta \mathrm{AC}: \mathrm{C})_{\text {sample }} /(\Delta \mathrm{AC}: \mathrm{C})_{\text {control }} \times 100$.

cell extracts. Sperm extracts from media supplemented with valine, isoleucine, methionine or heptanoate did not show any accumulation of propionylcarnitine, despite the fact that their catabolic pathways are reported (McGilvery \& Goldstein, 1979) to result in production of propionylCoA. Threonine appeared to produce a very small increase in propionylcarnitine content of cell extracts. Supplementation with isoleucine and leucine, whose catabolic pathways include production of methylbutyrylCoA and isovalerylCoA, respectively, did not cause the corresponding acylcarnitines to appear in sperm extracts. None of the above compounds affected sperm motility appreciably. Acetylcarnitine was therefore the major acylcarnitine in the spermatozoa under all the conditions studied and propionylcarnitine content was negligible by comparison. The remainder of the investigation therefore reports the acylated carnitine content of human spermatozoa as essentially entirely acetylcarnitine.

\section{Effect of substrates on acetylcarnitine:carnitine ratio}

The effect on carnitine acylation produced by incubating washed spermatozoa in the presence of substrates known to be present in seminal fluid was then tested. Results of a typical experiment (Table 3) show that pyruvate but not citrate or sorbitol resulted in a substantial increase of the acetylcarnitine:carnitine ratio. The percentage difference in this ratio between control and test 
Table 4. The effects produced on acetylcarnitine (AC):carnitine (C) ratios of washed human spermatozoa by incubation in the presence of exogenous substrate

\begin{tabular}{|c|c|c|c|c|c|}
\hline Substrate & $\begin{array}{l}\text { No. of } \\
\text { replicates }\end{array}$ & $\begin{array}{c}\text { Acetyl- } \\
\text { carnitine:carnitine }\end{array}$ & $\triangle \mathrm{AC}: \mathrm{C}^{*}$ & $\begin{array}{c}\% / \% \\
\text { Difference* }\end{array}$ & Significance $\dagger$ \\
\hline Fructose & 13 & $3 \cdot 7 \pm 1 \cdot 3$ & $0 \cdot 14 \pm 0 \cdot 50$ & $7 \cdot 7 \pm 15.6$ & n.s. \\
\hline Lactate & 8 & $5.4 \pm 1.9$ & $2.64 \pm 1.50$ & $96.2 \pm \quad 54.8$ & $P<0.005$ \\
\hline \multicolumn{6}{|l|}{ Lactate +} \\
\hline fructose & 4 & $4 \cdot 5 \pm 1 \cdot 3$ & $1.62 \pm 1.00$ & $55 \cdot 3 \pm 27 \cdot 1$ & $P<0.05$ \\
\hline Pyruvate & 16 & $6 \cdot 4 \pm 2 \cdot 1$ & $2.65 \pm 1.22$ & $71.9 \pm 27.8$ & $P<0.001$ \\
\hline \multicolumn{6}{|l|}{ Pyruvate +} \\
\hline fructose & 3 & $7 \cdot 0 \pm 2 \cdot 6$ & $3 \cdot 16 \pm 3 \cdot 70$ & $111 \cdot 3 \pm 148 \cdot 2$ & \\
\hline Sorbitol & 6 & $1.8 \pm 0.6$ & $-0.73 \pm 0.60$ & $-26 \cdot 0 \pm 14 \cdot 1$ & $P<0.01$ \\
\hline Citrate & 6 & $2.4 \pm 0.5$ & $-0.34 \pm 1.00$ & $-3 \cdot 1 \pm 33 \cdot 2$ & n.s. \\
\hline D-Glucose & 5 & $2 \cdot 1 \pm 0 \cdot 6$ & $-0.01 \pm 0.17$ & $-0.9 \pm 8.6$ & n.s. \\
\hline L-Aspartate & 8 & $4 \cdot 1 \pm 1 \cdot 6$ & $0.80 \pm 0.58$ & $24.2 \pm 11.2$ & $P<0.001$ \\
\hline Malate & 3 & $3 \cdot 4 \pm 1 \cdot 8$ & $-0.38 \pm 0.75$ & $-10 \cdot 7 \pm 16 \cdot 1$ & n.s. \\
\hline Acetate & 7 & $3 \cdot 4 \pm 1 \cdot 5$ & $0.17 \pm 0.37$ & $5.3 \pm 8.8$ & n.s. \\
\hline
\end{tabular}

Values are mean \pm s.d.

* See Table 3.

$\dagger$ Paired $t$ test; n.s., not significant.

samples was the value used to test the statistical significance of the effect of a substrate in a series of experiments because it best expressed the change in acylation in relationship to the control. The results of a series of similar experiments (Table 4) showed that pyruvate and lactate increased the acetylcarnitine:carnitine ratio in the spermatozoa dramatically. The effect of lactate was still evident when both lactate and fructose were present in the incubation mixture, but fructose alone did not affect it. Other substrates that were tested included aspartate, which increased the ratio slightly, sorbitol which decreased it, and acetate, citrate, succinate, malate and glucose which had no significant effect.

The above experiments indicated that pyruvate and lactate increased the acetylcarnitine: carnitine ratio, which had been shown to be low in immotile cells (Golan et al., 1984). To test whether that ratio could be increased in immotile cells (with possible improvement of their motility), viable but immotile spermatozoa were incubated in medium containing pyruvate or lactate. This treatment did not increase the acetylcarnitine:carnitine ratio of these cells or improve their motility.

\section{Discussion}

In previous reports from this laboratory we have demonstrated the presence of the acetyl-, propionyl-, butyryl-, isobutyryl- and isovalerylesters of carnitine in human semen (Lewin, Holzman, Fahimi, Choi \& Bieber, 1979). Spermatozoa themselves contained acetylcarnitine and traces of propionylcarnitine (Golan et al., 1984). All of these esters are produced in the reaction: acylCoA + L-carnitine $\rightleftharpoons$ acylcarnitine $+\mathrm{CoA}$, which is catalysed by carnitine acetyltransferase, an enzyme known to be present at high activity in spermatozoa (Brooks, 1979). Carnitine acetyltransferase is most active with acetylCoA as substrate but has declining activity with CoA esters of longer carbon chain lengths (Bieber \& Farrell, 1983). Substrates which can be converted into these CoA esters by spermatozoa might serve as sources of the corresponding carnitine esters. Bioautographic results showed that spermatozoa incubated in the presence of propionate showed increased content of propionylcarnitine. Incubation with acids of 4 and 5 carbon chain length did not result in the appearance of corresponding acylcarnitines, although the 5-carbon straightchain fatty acid, $n$-valerate, caused an increase in propionylcarnitine in the spermatozoa. This is 
consistent with its catabolism by beta-oxidation to propionylCoA and acetylCoA, the former being a precursor of propionylcarnitine. Incubation of spermatozoa in the presence of the amino acids valine, methionine or isoleucine (the catabolism of which should lead to propionylCoA production) did not result in increased propionylcarnitine in spermatozoa, and threonine caused only a trivial increase. In all cases acetylcarnitine was the major acylated form of carnitine in spermatozoa, confirming previous reports (Casillas \& Erickson, 1975; Golan et al., 1984). It was concluded, therefore, that the major role of the carnitine acetyltransferase system of human spermatozoa involves transfer of acetyl groups.

The equilibrium constant for the reaction: acetylcarnitine $+\operatorname{CoA} \rightleftharpoons$ acetylCoA + carnitine can be calculated to be:

$$
K=\frac{(\text { acetylCoA })(\text { carnitine })}{(\mathrm{CoA})(\text { acetylcarnitine })}, \text { or } \frac{(\text { acetylCoA })}{(\mathrm{CoA})}=\frac{K(\text { acetylcarnitine })}{(\text { carnitine })},
$$

where Fritz, Schultz \& Srere (1963) have determined that $K=0.6$. This equation shows that the equilibrium ratio of acetylcarnitine:carnitine is proportional to the corresponding ratio for coenzyme A. Casillas \& Erickson (1975) suggested that the carnitine acetyltransferase system of spermatozoa may function as a buffer for the acetylCoA:CoA ratio. We have previously reported that the acetylcarnitine:carnitine ratio of motile human spermatozoa is greater than that of immotile spermatozoa (Golan et al., 1984), indicating that variations in this ratio may be significant in regulating sperm motility. Johansen \& Bøhmer (1979) have also reported that the acetylcarnitine content of motile spermatozoa is greater than in immotile spermatozoa.

Substrates whose catabolic pathways produce or consume acetylCoA or CoA might be expected to influence the acetylcarnitine:carnitine ratio. When human spermatozoa were incubated in the absence of added external substrate the acetylcarnitine:carnitine ratio remained approximately constant for a period of at least $4 \mathrm{~h}$. The major endogenous energy source for bovine or ram spermatozoa has been shown to be fatty acid derived from cellular lipids (Mann, 1964). The major energy-yielding substrates present in human seminal fluid are fructose, sorbitol, pyruvate and lactate (Mann, 1964). Of these, only pyruvate and lactate increased the acetylcarnitine: carnitine ratio significantly. It is probable that they did so by producing acetylCoA via the pyruvic dehydrogenase reaction. Neither lactate nor pyruvate caused such an increase in the ratio in immotile cells, indicating that these cells did not utilize the substrates in the same manner as did motile spermatozoa. Pyruvate and lactate are found in fluids of the female reproductive tract (Hamner, 1973; Moghissi, 1973) and they are routinely added to medium for sperm capacitation and in-vitro fertilization. Sorbitol may be oxidized to fructose and aspartate may be deaminated to oxaloacetate, but the explanation for the effect of these compounds on the acetylcarnitine:carnitine ratio is not apparent.

In studies with bull and monkey spermatozoa, Casillas \& Erickson (1975) observed substrateinduced elevations in the acetylation state of carnitine caused by incubation with a wide variety of substrates including fructose, glucose, acetate, pyruvate and lactate. In our studies with human spermatozoa the medium of Casillas \& Erickson (1975) did not support progressive motility for an extended period unless albumin was added. In the presence of albumin fructose did not cause an increase in the acetylcarnitine:carnitine ratio. Albumin apparently exerts subtle effects on the cell membrane which affect both the motility and the ability to regulate the acetylcarnitine:carnitine ratio.

Further experiments are in progress to clarify the relationships between carnitine, coenzyme A and pyruvate metabolism and motility in spermatozoa.

This project was supported in part by a grant to R.G. from the Schreiber Fund, Tel Aviv University. We thank Ms Juliana Ben Zimra for performing semen analyses and Mrs Sonia Cojocaru for excellent secretarial assistance. 


\section{References}

Bieber, L.L. \& Farrell, S. (1983) Carnitine acyltransferases. In The Enzymes, 3rd edn, vol. 16, pp. 627-644. Ed. P. D. Boyer. Academic Press, New York.

Brooks, D.E. (1979) Carnitine, acetylcarnitine and the activity of carnitine acyltransferases in seminal plasma and spermatozoa of men, rams and rats. $J$. Reprod. Fert. 56, 667-673.

Casillas, E.R. (1973) Accumulation of carnitine by bovine spermatozoa during maturation in the epididymis. J. biol. Chem. 248, 8227-8232.

Casillas, E.R. \& Erickson, B.J. (1975) The role of carnitine in spermatozoon metabolism: substrate-induced elevation in the acetylation state of carnitine and coenzyme $\mathbf{A}$ in bovine and monkey spermatozoa. Biol. Reprod. 12, 275-283.

Cederblad, G. \& Lindstedt, S. (1972) A method for the determination of carnitine in the picomole range. Clin. chim. Acta 37, 235-243.

Freund, M. \& Peterson, R.N. (1976) Semen evaluation and fertility. In Human Semen and Fertility Regulation in Men, pp. 344-354. Ed. E. S. E. Hafez. C. V. Mosby, St Louis.

Fritz, I.B., Schultz, S.K. \& Srere, P.A. (1963) Properties of partially purified carnitine acetyltransferase. $J$. biol. Chem. 238, 2509-2517.

Golan, R., Weissenberg, R. \& Lewin, L.M. (1984) Carnitine and acetylcarnitine in human motile and immotile spermatozoa. Int. J. Androl. 7, 484494.

Gorus, F.K., Finsy, R. \& Pipeleers, D.G.C. (1982) Effect of temperature, nutrients, calcium and cAMP on motility of human spermatozoa. Am. J. Physiol. 242, c304-c311.

Hamner, C.E. (1973) Oviducal fluid, composition and physiology. In Handbook of Physiology, Sec. 7, Vol. II, pp. 141-151. Eds R. O. Greep \& E. B. Astwood. Amer. Physiol. Soc., Washington, D.C.

Harrison, R.A.P. (1976) A highly efficient method for washing mammalian spermatozoa. J. Reprod. Fert. 48, 347-353.
Hinton, B.T., Brooks, D.E., Dott, H.M. \& Setchell, B.P. (1981) Effects of carnitine and some related compounds on the motility of rat spermatozoa from the caput epididymidis. J. Reprod. Fert. 61, 59-64.

Hutson, S.M., Van Dop, C. \& Lardy, H.A. (1977) Mitochondrial metabolism of pyruvate in bovine spermatozoa. J. biol. Chem. 252, 1309-1315.

Jeulin, C., Soufir, J.C. \& Jouannet, P. (1981) Effects of L-carnitine and D,L-acetylcarnitine on human sperm motility as measured by laser Doppler velocimetry. IRCS Medical Sci. 9, 722-723.

Johansen, L. \& Bøhmer, T. (1979) Motility related to carnitine/acetylcarnitine in human spermatozoa. Int. $J$. Androl. 2, 202-210.

Lewin, L.M. \& Bieber, L.L. (1979) Paper chromatography and bioautography of L-carnitine and its acyl esters. Analyt. Biochem. 96, 322-325.

Lewin, L.M., Holzman, G., Fahimi, F., Choi, Y. \& Bieber, L.L. (1979) Carnitine acyl esters of human semen. Int. J. Androl. 2, 542-548.

Lewin, L.M., Orenstein, H., Nebel, L. \& Emaus, R. (1981) Bioautography of carnitine derivatives: Compounds in human semen possessing biological activity for a carnitine-requiring mutant of the yeast Torulopsis bovina. Clin. Biochem. 14, 305-308.

Mann, T. (1964) Biochemistry of Semen and of the Male Reproductive Tract. Methuen \& Co., London.

McGilvery, R.W. \& Goldstein, G. (1979) Biochemistry, a Functional Approach, 2nd edn, pp. 565-588. W. B. Saunders \& Co., Philadelphia.

Moghissi, K.S. (1973) Composition and function of cervical secretion. In Handbook of Physiology, Sec. 7, Vol. II, pp. 25-48. Eds R. O. Greep \& E. B. Astwood. Amer. Physiol. Soc., Washington, D.C.

Tanphaichitr, N. (1977) In vitro stimulation of human sperm motility by acetylcarnitine. Int. J. Fertil. 22, 85-91. 